\title{
Patterns of blood-brain barrier impairment and clinical features in multiple sclerosis
}

\author{
B N McLean, A Z J Zeman, D Barnes, E J Thompson
}

\begin{abstract}
Seventy four patients with clinically definite multiple sclerosis were studied by using polyacrylamide gel electrophoresis of cerebrospinal fluid to assess bloodbrain barrier function. Blood-brain barrier impairment was associated with recent clinical relapses of multiple sclerosis and worsened across a spectrum from the relapsing-remitting type of multiple sclerosis to secondary and primary progressive disease. The association between blood-brain barrier impairment and primary progressive disease is particularly interesting in the light of recent evidence that focal gadolinium enhancement on MRI is relatively unusual in patients with this disease.
\end{abstract}

(F Neurol Neurosurg Psychiatry 1993;56:356-360)

Focal disruption of the blood-brain barrier is a well established feature of the lesions of multiple sclerosis. Broman showed that trypan blue, which is usually excluded by the blood-brain barrier escapes from cerebral vessels in the vicinity of plaques after death. ${ }^{1}$ More recent histopathological studies have illustrated the occurrence of a protein rich exudate from veins and capillaries in acute plaques and have emphasised that the ring of leakage extends deeply into normally myelinated white matter around the edge of plaques. ${ }^{23}$ These pathological observations are in keeping with evidence from neuroimaging, from both contrast CT scanning ${ }^{4}$ and gadolinium enhanced MRI, ${ }^{5}$ that increased permeability of the blood-brain barrier to contrast agents is a consistent early feature of new lesions in multiple sclerosis.

Several lines of evidence suggest that this impairment of the blood-brain barrier may play a part in the pathogenesis both of the remitting and of the persistent neurological disability caused by multiple sclerosis. Firstly, the use of high dose intravenous steroids, which has been shown to accelerate recovery from relapse, is associated with an appreciable reduction in both contrast CT scan and gadolinium MRI enhancement of recent lesions. ${ }^{678}$ This effect, with concomitant reduction of oedema and of serum protein leakage, may be related to the short term therapeutic effect of steroids. Secondly, the study of experimental allergic encephalo- myelitis, ${ }^{9}$ and limited clinico pathological correlation in multiple sclerosis, ${ }^{10}$ suggest that the focal blood-brain barrier impairment detected by gadolinium MRI in these conditions is accompanied by perivascular inflammation. Work in vitro with oligodendrocyte and other glial cell lines has shown the selective vulnerability of oligodendrocytes to damage by exposure to cytokines ${ }^{11}$ and serum proteins, for example complement. ${ }^{12}$ The combination of perivascular inflammation and associated focal breakdown in the bloodbrain barrier might be expected to cause focal demyelination and tissue damage of the kind seen in the established lesions of multiple sclerosis.

Study of the CSF, which intercommunicates freely with brain extracellular fluid, ${ }^{13}$ offers another approach to the investigation of blood-brain barrier disturbance in multiple sclerosis. Albumin concentrations in CSF are raised in only a fifth of patients with multiple sclerosis, ${ }^{14}$ and measurement of albumin or total protein is a relatively insensitive gauge of blood-brain barrier impairment. Polyacrylamide gel electrophoresis of CSF is a well established technique, used routinely in many laboratories, principally for detecting oligoclonal bands. ${ }^{1516}$ It is also a sensitive tool in the assessment of blood-brain barrier function for detecting subtle changes in the distinctive protein profile of normal CSF resulting from a relatively minor influx of serum across a damaged blood-brain barrier. ${ }^{15} 1718-19$

Using this approach Takeoka et al showed that, among patients with multiple sclerosis and raised total protein in CSF, qualitative electrophoretic abnormalities suggestive of blood-brain barrier disturbance were detectable in $95 \%$ of patients; in those patients with multiple sclerosis whose total protein was normal, $56 \%$ nevertheless displayed qualitative abnormalities. ${ }^{19}$ No distinctions were made in this work between different clinical subtypes of multiple sclerosis, nor between patients in relapse and those in remission. Walker et al found that blood-brain barrier impairment, defined by abnormalities, on polyacrylamide electrophoresis, was more common among patients with primary and secondary progressive multiple sclerosis than among patients with relapsing-remitting disease. ${ }^{20}$

The work reported here extends these findings by describing the relations between two grades of blood-brain barrier disturbance 
(mild and moderate), as assessed by polyacrylamide gel electrophoresis, and a number of clinical features of multiple sclerosis: clinical type (relapsing-remitting, primary and secondary progressive), recency of relapse, and degree of disability. Our principal aims were to examine the pattern of blood-brain barrier impairment in relapse and remission and to discover whether analysis of CSF can distinguish the clinical types of multiplesclerosis.

\section{Methods}

CLINICAL

The study population consisted of consecutive patients with clinically definite multiple sclerosis who underwent lumbar puncture in the course of the routine clinical investigation of their symptoms between 1986 and 1988 . All were inpatients at the National Hospital for Neurology and Neurosurgery, and full neurological examinations were documented in every case. In many cases Kurtzke disability scores $^{22}$ had been recorded at the time of the examination.

Four categories of information were obtained retrospectively from the clinical notes. (i) Clinical type of disease: relapsingremitting disease was defined as disease with relapses followed by substantial or complete remissions; secondary progressive disease was defined as disease that was initially relapsingremitting, but in which a progressive worsening had supervened, with or without continuing superimposed relapses; primary progressive disease was defined as disease progressive from onset, without clinical evidence of relapse, but with evidence for new lesions appearing at least one month from onset. ${ }^{21}$ Patients' disease types were classified from the clinical notes by one of the authors (BM), and then checked independently by another (DB) who was blind to the laboratory findings. (ii) Date of last relapse: relapse was defined as the development of a neurological disturbance, or worsening of a pre-existing disturbance, lasting for at least 24 hours. Patients within two months of the onset of a relapse were arbitrarily taken to be "in relapse." (iii) Date of lumbar puncture, and therefore proximity to relapse. (iv) Kurtzke disability score ${ }^{22}$ at the time of the lumbar puncture. Most patients had been scored during their admission, and in these cases the assessment was checked by one of the authors; the remaining patients were scored retrospectively: where the notes did not allow confident scoring of disability the patients were excluded from further study.

BLOOD-BRAIN BARRIER IMPAIRMENT

Total protein in CSF was measured by using benzethonium chloride, as previously described. ${ }^{23}$ Albumin and IgG concentrations were determined by electroimmunoassay. ${ }^{24} \mathrm{~A}$ volume of CSF containing a standard protein loading of $100 \mu \mathrm{g}$ was then electrophoresed on $6 \mathrm{~mm}$ rod polyacrylamide gels, made up to $7 \% \mathrm{~T}, 2.5 \% \mathrm{C}$, as previously described..$^{25}$ After electrophoresis, gels were stained with Coomassie brilliant blue and destained with $7 \%$ acetic acid. The gels were then scanned with a microdensitometer (Joyce Loeble Chromoscan 3) to measure the staining density of the following protein bands: group components ( $\mathrm{Gc1}$ and $\mathrm{Gc2}$ ), haptoglobin

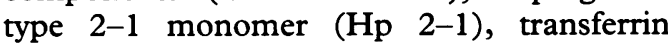
(Trf), and asialotransferrin protein (also known as "tau" protein). The distance travelled by albumin along the gel was taken as one unit; the relative distances travelled by the bands of interest, at which they were scanned by the microdensitometer, were 0.32 (Hp 2-1);0.53 (Trf); 0.76 (Gc2); and 0.82 (Gc1). The microdensitometer generated a printout displaying automated measurements of the peak absorbance of the protein bands, both above protein free background ("height") and above the immediately preceding region of gel ("size"). All gels were also inspected by eye to ensure that the densitometric measurements were of the correct protein bands.

CSF samples containing more than 100 red blood cells $/ \mathrm{mm}^{3}$ or with haptoglobinhaemoglobin complexes in the ' $\gamma$ ' region, were excluded from the study because of the effect of contamination by blood on the density and position of the $\mathrm{Hp} \mathrm{2-1}$ band. The greater of the two height values of the group components was taken as Gc height for the purposes of the definitions below.

Three categories of blood-brain barrier function were defined: (i) normal: CSF samples in which the ratio of $\mathrm{Hp} \mathrm{2-1}$ size to Trf height was $<0.01$ and in which the ratio of $\mathrm{Gc}$ height to tau height was $<1$; (ii) mild impairment: samples in which the ratio of $\mathrm{Hp}$ $2-1$ size to $\operatorname{Trf}$ height was $>0.01$, but in which the ratio of $\mathrm{Gc}$ height to tau height was $<1$; (iii) moderate impairment: samples in which the ratio of $\mathrm{Gc}$ height to tau height was $>1$. These distinctions are based upon a previous study of over 1000 specimens ${ }^{19}$ and are in basic agreement with the data of Felgenhauer. ${ }^{26}$ We chose to distinguish only two categories of blood-brain barrier impairment, mild and moderate, as extreme disruption of the blood-brain barrier with CSF total protein of more than $1 \mathrm{~g} / \mathrm{L}$ is rarely seen in multiple sclerosis and is indeed sufficiently unusual to cast some doubt upon the diagnosis. ${ }^{27}$

\section{STATISTICS}

Relations between blood-brain barrier function and clinical type of disease were analysed with Spearman's rank correlation. The $\chi^{2}$ test and Fisher's exact test were used to examine the relation between blood-brain barrier function and recency of relapse. The KruskalWallis test for non-parametric data was used to analyse differences in age, disability, disease duration, albumin quotients, and IgG indices between the three clinical types of disease; a corrected Mann-Whitney test was used to probe pairwise differences between the types. 
Table 1 Clinical details of the study patients.

\begin{tabular}{llll}
\hline & \multicolumn{2}{l}{ Disease type } & \\
\cline { 2 - 4 } & $\begin{array}{l}\text { Primary } \\
\text { progressive }\end{array}$ & $\begin{array}{l}\text { Relapsing- } \\
\text { remitting }\end{array}$ & $\begin{array}{l}\text { Secondary } \\
\text { progressive }\end{array}$ \\
\hline Total No & $(22)$ & $(26)$ & $(26)$ \\
\hline No of men & 11 & 11 & 13 \\
Mean age & 50 & 37 & 45 \\
Mean duration of disease (years) & 7 & 6 & 14 \\
Mean disability (Kurtzke score) & 4.5 & 2.0 & 4.0 \\
\hline
\end{tabular}

\section{Results}

PATIENT DETAILS

Seventy four patients were included, 35 men and 39 women. Numbers, sex ratios, ages, disease durations, and disability scores in the three clinical groups are summarised in table 1. There were significant differences between the three groups with respect to age, disease duration, and disability $(p<0.01)$. Patients with relapsing-remitting disease were on average younger than patients in the other two groups. Patients with secondary progressive disease had a longer duration of disease than patients in the other two groups. Patients in the primary and secondary progressive groups were significantly more disabled than those in the relapsing-remitting group. The sex ratios did not differ significantly between groups. A total of 67 of the patients $(91 \%)$ had oligoclonal bands of IgG in their CSF, but not in their serum, on isoelectric focusing.

\section{BLOOD-BRAIN BARRIER IMPAIRMENT AND CLINICAL TYPES}

Table 2 summarises the results for bloodbrain barrier function in the three clinical types of multiple sclerosis. There was a significant tendency for function to worsen from relapsing-remitting through secondary progressive to primary progressive disease $(r=$ $0.38, p=0.003)$. This trend was more pronounced when patients in relapse in the relapsing-remitting and secondary progressive groups were excluded from the analysis $(r=0.52, p=0.0001 ;$ table 2$)$.

Neither albumin quotients (CSF albumin/ serum albumin) nor IgG indices (CSF IgG/serum IgG)/(CSF albumin/serum albumin) distinguished the three groups, including or excluding patients in relapse. The albumin quotient was significantly higher in patients with moderate blood-brain dysfunction (mean $1.2 \times 10-2$ ) than in patients with normal function (mean $0.57 \times 10-2$; $\mathrm{p}<0.05)$.

Table 2 Blood brain barrier function and disease types. Values are numbers (percentages)

\begin{tabular}{|c|c|c|c|}
\hline \multirow{2}{*}{$\begin{array}{l}\text { Blood-brain } \\
\text { barrier } \\
\text { function }\end{array}$} & \multicolumn{3}{|l|}{ Disease type } \\
\hline & $\begin{array}{l}\text { Relapsing- } \\
\text { remitting }\end{array}$ & $\begin{array}{l}\text { Secondary } \\
\text { progressive }\end{array}$ & $\begin{array}{l}\text { Primary } \\
\text { progressive }\end{array}$ \\
\hline $\begin{array}{l}\text { Normal } \\
\text { Mild impairment } \\
\text { Moderate impairment } \\
\text { Total }\end{array}$ & $\begin{array}{rr}21 & (81) \\
4 & (15) \\
1 & (4) \\
26 & (100)\end{array}$ & $\begin{array}{l}\text { ing patients } i \\
18(69) \\
4(15) \\
4(15) \\
26(100)\end{array}$ & $\begin{aligned} & 7(32) \\
& 11(50) \\
& 4(18) \\
& 22(100)\end{aligned}$ \\
\hline $\begin{array}{l}\text { Normal } \\
\text { Mild impairment } \\
\text { Moderate impairment } \\
\text { Total }\end{array}$ & $14(100)$ & $\begin{array}{l}\text { ling patients } \\
18(78) \\
4 \quad(17) \\
1 \\
23(100)\end{array}$ & $\begin{aligned} 7 & (32) \\
11 & (50) \\
4 & (18) \\
22 & (100)\end{aligned}$ \\
\hline
\end{tabular}

The quotients among patients with moderate and mild (mean $0.64 \times 10-2)$ dysfunction were not significantly different.

\section{BLOOD-BRAIN BARRIER IMPAIRMENT AND}

RECENCY OF RELAPSE

Among patients susceptible to relapse, in the relapsing-remitting and secondary progressive groups, impairment was more common in relapse than in remission $(p<0.0001)$. Indeed, in the relapsing-remitting group, only patients in relapse had evidence of bloodbrain barrier impairment.

\section{BLOOD-BRAIN BARRIER IMPAIRMENT AND}

DISABILITY

There was a trend towards an association between increasing blood-brain barrier impairment and increasing disability but this did not attain significance.

\section{Discussion}

Our main findings are that the state of the blood-brain barrier, as judged by CSF electrophoresis of cerebrospinal fluid, differed significantly between the clinical types of multiple sclerosis and worsened in relapse. As would be expected, our patients with primary and secondary progressive disease were more disabled and older than patients with the relapsing-remitting type. Disease duration was longer among patients with secondary progressive multiple sclerosis than in the other two groups.

The association between recent relapse and blood-brain barrier impairment, is in keeping with a number of reports that other CSF variables reflect the occurrence of recent relapse: thus, following a relapse, there is a trend towards a higher CSF leucocyte count, ${ }^{20}$ an increased likelihood of finding free light chains of immunoglobulin, ${ }^{28}$ and an increased incidence of oligoclonal $\operatorname{IgM} .^{29}$

The association between primary progressive disease and blood-brain barrier impairment is consistent with a previous result from this laboratory ${ }^{20}$ but is perhaps unexpected in the context of recent MRI findings. In Thompson et al's patients, new lesions were less often seen on MRI, and less frequently enhanced with gadolinium, in primary than in secondary progressive disease, despite similar levels of disability and rates of disease progression. ${ }^{30}$ There are several possible explanations for this discrepancy. It may be that the lesions underlying the blood-brain barrier impairment which we have detected in primary progressive multiple sclerosis are smaller, or more diffuse; undergo blood-brain barrier disturbance of briefer duration; or are more often spinal than those of secondary progressive multiple sclerosis. Alternatively, a predilection for involvement of the spinal cord in primary progressive disease might give rise to an unrepresentative effect on the protein composition of lumbar CSF. As Thompson et al suggest, comparative pathological study of patients with primary and sec- 
ondary progressive multiple sclerosis may help to resolve this issue. ${ }^{30}$

Several variables may be interacting to produce the trend we have reported here for blood-brain barrier impairment to worsen across a spectrum from relapsing-remiting to secondary and primary progressive disease: age, disease duration, disability, immobility (which may be associated with a rise in CSF total protein ${ }^{31}$ ), and clinical type. However, our data suggest that the primary association is between blood-brain barrier impairment and clinical type of disease. This emerges particularly clearly from a comparison of the primary and secondary progressive groups: age and degree of disability were similar in these two groups, and disease duration was greater in the secondary progressive group, yet blood-brain barrier impairment was significantly greater among the primary progressive patients than among the secondary progressive patients.

Our results must be evaluated in relation to the disadvantages of a relatively small and partly retrospective study and the interpretation of the electrophoretic findings used to assess blood-brain barrier integrity. These will be discussed in turn.

Retrospective assessment of clinical notes may lead to imprecision in the quantification of disability, the assignment of clinical type and the timing of relapses. However, provided, as in this study, the assessor is blind to the results with which the clinical features are to be correlated, retrospective analysis should not introduce any systematic bias, although it may of course obscure important findings, by contaminating the results with excessive "noise".

The principle underlying our method of assessment of the blood-brain barrier is that, as the barrier is breached the CSF, which has a distinctive protein profile, comes to resemble serum more closely than is usual. ${ }^{1517-1926}$ In the early stages of blood-brain barrier impairment the approximation of the CSF protein profile to that of serum can occur while total protein is still within the normal range. ${ }^{1719}$ The failure of the albumin quotient to distinguish clinical groups that were clearly distinct on CSF electrophoresis is in keeping with this observation.

Mild blood-brain barrier impairment is characterised by the appearance of haptoglobin species (Hp type 2-1 monomer and types 2-1 and 2-2 polymers) which are normally largely excluded by the intact barrier. ${ }^{17} 19263233$ One disadvantage of our method for detecting mild impairment, which depends on measurement of the amount of $\mathrm{Hp} \mathrm{2-1} \mathrm{monomer,}$ is that the $\mathrm{Hp} \mathrm{2-1}$ species can only be found in the CSF of individuals with the 2-1 haptoglobin genotype; this is the commonest of the haptoglobin genotypes in European populations, but "mild" impairment can thus only be detected in about half of individuals. ${ }^{34}$ Although this will result in an underestimate of the prevalence of mild blood-brain barrier impairment in all groups of patients, we are not aware of any evidence that there is an association between clinical type of multiple sclerosis and haptoglobin haplotype; thus we would expect any inaccuracy in the estimate of the frequency of mild impairment to be uniform across groups. A second consideration is that no published large scale electrophoretic studies show that concentrations of Hp 2-1 monomer are consistently low in normal CSF, although the available evidence from smaller studies suggests this. ${ }^{1733}$

Is impairment of the blood-brain barrier the only possible explanation of the association between the presence in CSF of the $\mathrm{Hp}$ 2-1 monomer and the clinical features described in this paper? We believe that it is the most likely explanation, and it is in keeping with the finding that moderate bloodbrain barrier impairment is associated with the same clinical features; alternative possibilities include an increase in serum levels of $\mathrm{Hp}$ 2-1 monomer, ${ }^{35}$ and a genetic association between the $\mathrm{Hp}$ 2-1 genotype and disease type in multiple sclerosis. We hope to analyse these possibilities further in future work.

The detection of "moderate" impairment of the blood-brain barrier is less controversial and depends upon the fact that a protein specific to CSF ("tau" protein, a desialated derivative of transferrin) is progressively "diluted out" as serum proteins enter the CSF across an impaired barrier. ${ }^{15} \mathrm{~A}$ ratio of group components to tau protein greater than 1 is generally associated with a raised total concentration of CSF protein: this is born out in the present results by the raised albumin quotient in patients with moderate bloodbrain barrier impairment.

Thus this study of associations between the state of the blood-brain barrier, as assessed from the CSF, and clinical features in multiple sclerosis suggests that clinical relapse and progressive, especially primary progressive, disease are associated with breakdown of the barrier. These findings may be of pathogenetic importance with respect to the access of myelinotoxic factors to the central nervous system, particularly in patients with primary progressive disease, in whom disability tends to be greatest. They provide further rationale for treatment aimed at stabilising the bloodbrain barrier. A prospective study is underway to explore these findings further, and to establish whether evidence of blood-brain barrier impairment from the CSF predicts a response to treatment with intravenous steroids. Neuropathological study of tissue from patients with differing disease types may help to clarify the anatomical basis of these findings.

We thank Mr Peter Sacares for statistical advice.

1 Broman T. Blood brain barrier damage in multiple sclerosis: supravital test observations. Acta Neurol Scand 1964;21-4.

2 Adams CMW, Poston RN, Buk SJ, Sidhu YS, Vipod H. Inflammatory vasculitis in multiple sclerosis. 7 Neurol Sci 1985;69:269-83.

3 Gay D, Esiri M. Blood-brain barrier damage in acute multiple sclerosis plaques. Brain 1991;114:557-72.

4 Lebow S, Anderson DC, Mastri A, Larson D. Acute multiple sclerosis with contrast enhancing plaques. Arch Neurol 1978;35:439-9. 
5 Kermode AG, Thompson AJ, Tofts PS, et al. Breakdown of the blood-brain barrier precedes symptoms and other MRI signs of new lesions in multiple sclerosis: pathogenetic and clinical implications. Brain 1990;113 1477-89.

6 Toriano R, Hafstein M, Ruderman M, Dowling P, Cook $S$. Effect of high dose intravenous steroid administration on contrast enhancing computerised tomographic scan lesions in multiple sclerosis. Ann Neurol 1984;15: 257-63.

7 Barkhof F, Frequin STFM, Hommes OR, et al. A correlative triad of gadolinium-DTPA MRI, EDSS and CSFMBP in relapsing multiple sclerosis patients treated with high dose intravenous methylprednisolone. Neurology high dose intrav $1992 ; 42: 63-7$.

8 Miller DH, Thompson AJ, Morrisey SP, et al. High dose steroids in acute relapses of multiple sclerosis: MRI evidence for a possible mechanism of therapeutic effect. f Neurol Neurosurg Psychiatry 1992;55:450-3.

9 Hawkins CP, Munro PMG, Mackenzie F, Kesselring J, Tofts PS, Du Bouley EPGH, et al. Duration and selectivity of blood-brain barrier breakdown in chronic relapsing experimental allergic encephalomyelitis studied by gadolinium-DTPA and protein markers. Brain 1990;113:365-78.

$10 \mathrm{Katz}$ D, Taubenberger J, Raine C, McFarlin D, McFarland H. Gadolinium-enhancing lesions on magnetic resonance imaging: neuropatholigical findings. Antic resonance imaging:

11 Selmaj K, Raine CS, Cannella B, Brosnan CF Identification of lymphotoxin and tumor necrosis facto in multiple sclerosis lesions. F Clin Invest 1991;87 949-54.

12 Compston DAS. The dissemination of multiple sclerosis. $f R$ Coll Phys 1990;24:207-18.

13 Davson K, Welch K, Segal MB. The physiology and pathophysiology of the cerebrospinal fluid. Edinburgh: Churchill Livingstone, 1987; 146.

14 Tourtellotte W, Ma BI. Multiple sclerosis: the bloodbrain-barrier and the measurement of de novo central nervous system IgG synthesis. Neurology 1978;28: 76-83.

15 Thompson EJ, Johnson MH. Electrophoresis of CSF proteins. Br f Hosp Med 1982;28:600-8.

16 Chapel H, Haeney M. Essentials of clinical immunology. Oxford: Blackwell Scientific, 1988: 404-6.

17 Evans JH, Quick DT. Polyacrylamide gel electrophoresis of spinal fluid proteins. Arch Neurol 1966;14:64-72.

18 Johnson MH. A study of cerebrospinal fluid abnormalities in neurological disease [dissertation]. Oxford: University of Oxford, 1985.
19 Takeoka T, Shinohara Y, Furumi K, Mori K. Impairment of blood-cerebrospinal fluid barrier in multiple sclerosis. f Neurochem 1983;41:1102-8.

20 Walker RWH, Thompson EJ, McDonald WI Cerebrospinal fluid in multiple sclerosis: relationships between immunoglobulins, leucocytes and clinical features. 7 Neurol 1985;232:250-9.

21 Poser CM, Paty DW, Scheinberg L, et al. New diagnostic criteria for multiple sclerosis: guidelines for research protocols. Ann Neurol 1983;13:227-31.

22 Kurtzke JF. Further notes on disability evaluation in multiple sclerosis, with scale modifications. Neurology 1965;15:654-61.

23 Luxton RW, Patel P, Keir G, Thompson EJ. A micromethod for measuring total protein in cerebrospinal fluid by using benzethonium chloride in microtiter plate wells. Clin Chem 1989;35:1731-4.

24 Keir G, Thompson EJ. Proteins as parameters in the discrimination between different blood-CSF barriers. f Neurol Sci 1986;75:245-53

25 Thompson EJ. The cerebrospinal fluid proteins: a biochemical approach. Amsterdam: Elsevier, 1988: 138-44.

26 Felgenhauer K. Vergleichende Disc-elektrophorese von Serum and Liquor cerebrospinalis. Stuttgart: 1971, Thieme and
$1-62$.

27 Fishman RA. Cerebrospinal fluid in disorders of the nervous system. Philadelphia: WB Saunders, 1980: 309.

28 Vakaet A, Thompson EJ. Free light chains in the cerebrospinal fluid: an indicator of recent immunological stimulation. $f$ Neurol Neurosurg Psychiatry 1985;48 995-9.

29 Sharief $\mathrm{MK}$, Thompson EJ. Intrathecal immunoglobulin $M$ synthesis in multiple sclerosis. Brain 1991;114: 181-95.

30 Thompson AJ, Kermode AG, Wicks D, et al. Major differences in the dynamics of primary and secondary progressive multiple sclerosis. Ann Neurol 1991;29: 53-62.

31 Cumings $\mathrm{JN}$. The diagnostic value of the examination of cerebrospinal fluid. Postgrad Med $\mathcal{f}$ 1952;28:587-91.

32 Blau JN, Harris H, Robson EB. Haptoglobins in cere brospinal fluid. Clin Chim Acta 1963;8:202-6.

33 Takeoka T, Shinohara Y, Furumi K, Mori K Characteristic protein fractions of cerebrospinal fluid disc electrophoretic analysis. Brain $R$ 1980;198:147-56.

34 Putnam FW. Haptoglobin. In: The plasma proteins. Orlando: Academic Press, 1984.

35 Phillips SM, Kornguth SE, Thompson HG, Oldenburg J. Serum haptoglobin changes in multiple sclerosis. Neurology 1965;15:415-8. 